\title{
PARAMETER-DEPENDENT CORRECTED SIMPSON'S RULE
}

\author{
F. Dubeau \\ Mathematics Department \\ University of Sherbrooke \\ 2500 University Boul., Sherbrooke (Qc), CANADA, J1K 2R1
}

\begin{abstract}
We revisit the analysis of the endpoint corrected Simpson's quadrature rule. We present a parameter-dependent family of Euler-Maclaurin like formulae associated to Simpson's rule. Using Taylor's expansions and Peano's kernels we obtain optimal truncation error bounds. The parameter can be used to increase the order of the truncation error. The process can be applied to any interpolatory quadrature formulae.
\end{abstract}

AMS Subject Classification: 65B15, 65D30, 41A55

Key Words: Euler-Maclaurin Formula, corrected Simpson's rule, Taylor's expansion, Peano Kernel

\section{Introduction}

Any definite integral can be transformed as follows

$$
\int_{a}^{b} F(x) d x=\int_{-h}^{h} f(x) d x
$$

where $h=\frac{b-a}{2}$, and $f(x)=F\left(\frac{a+b}{2}+x\right)$, so we will consider the integral

$$
Q_{0}(f ; h):=\frac{1}{h} \int_{-h}^{h} f(x) d x
$$

to be approximated by quadrature rules. More precisely in this paper we start with the Simpson's rule and present a parameter-dependent family of Euler-

Received: $\quad$ October 4, 2016

Revised: December 13, 2016

Published: $\quad$ February 1, 2017 (c) 2017 Academic Publications, Ltd. url: www.acadpubl.eu 
Maclaurin like formulae

$$
\begin{aligned}
& \int_{-h}^{h} f(x) d x= \\
& h\left[\left(\frac{1}{3}-2 \lambda\right) f(-h)+\left(\frac{4}{3}+4 \lambda\right) f(0)+\left(\frac{1}{3}-2 \lambda\right) f(h)\right] \\
& +\sum_{k=1}^{K} h^{2 k} \gamma_{2 k}(\lambda)\left[f^{(2 k-1)}(h)-f^{(2 k-1)}(-h)\right]+o\left(h^{n(2 K, \lambda)+1}\right)
\end{aligned}
$$

where $\lambda$ is a real valued parameter. Moreover $\lambda$ can be choosen to encrease the degree of accuracy $n(2 K, \lambda)$ of the process. More precisely $n(2 K, \lambda)=2 K+1$ for any values of $\lambda$, except for one value $\lambda=\lambda_{2 K}$ for which $n\left(2 K, \lambda_{2 K}\right)=2 K+3$.

The method we propose to obtain these formulae is based on the degree of accuracy of the processes. We extend the approach proposed in [5, 7]. Our results include those presented in $[2,8]$, and the method could be extended and applied to other interpolatory quadrature rules [7]. Let us remark that corrected quadrature rules using derivatives at the endpoints of the interval of integration were studied before and are related to Euler-Maclaurin like formulae, see $[2,4,6,8,9]$ and references therein.

\section{Preliminaries}

\subsection{A Family of Integrals}

The basic integral we want to compute is

$$
Q_{0}(f ; h):=\frac{1}{h} \int_{-h}^{h} f(x) d x,
$$

and the endpoint correcting terms we are going to use are

$$
\begin{aligned}
Q_{2 k}(f ; h) & :=h^{2 k-1}\left[f^{(2 k-1)}(h)-f^{(2 k-1)}(-h)\right] \\
& =h^{2 k-1} \int_{-h}^{h} f^{(2 k)}(x) d x
\end{aligned}
$$

for $k=1,2,3, \ldots$ These expressions share the property

$$
Q_{2 k}\left(x^{l} ; h\right)=h^{l} Q_{2 k}\left(x^{l} ; 1\right)
$$


for $k=0,1,2, \ldots$, and $l=0,1,2, \ldots$ Hence we have

$$
Q_{0}(1 ; 1)=2,
$$

and, for $k \geq 1$ and $f(x)=x^{l}$,

$$
Q_{2 k}\left(x^{l} ; 1\right)=0
$$

for $l=0,1,2, \ldots, 2 k-1$. Also, for $l \geq 2 k \geq 0$ we have

$$
\begin{aligned}
Q_{2 k}\left(x^{l} ; 1\right) & =\frac{(2 k) !}{l+1}\left(\begin{array}{c}
l+1 \\
2 k
\end{array}\right)\left[1+(-1)^{l-2 k}\right] \\
& =\left\{\begin{array}{ccc}
0 & \text { for odd } l-2 k, \\
2 \frac{(2 k) !}{l+1}\left(\begin{array}{c}
l+1 \\
2 k
\end{array}\right) & \text { for even } l-2 k,
\end{array}\right.
\end{aligned}
$$

where we used the notation

$$
\left(\begin{array}{c}
m \\
n
\end{array}\right)=\frac{m !}{n !(m-n) !} \quad \text { for } \quad 0 \leq n \leq m .
$$

Hence $Q_{2 k}\left(x^{2 l+1} ; h\right)=0$ for $l=0,1,2, \ldots$

\subsection{Quadrature Rules}

To approximate $Q_{2 k}(f ; h)$, we consider symmetric quadrature rules of the form

$$
Q_{2 k}^{S i m}(f ; h)=a(k) f(-h)+b(k) f(0)+a(k) f(h),
$$

which have the following properties

$$
Q_{2 k}^{S i m}\left(x^{l} ; h\right)=h^{l} Q_{2 k}^{S i m}\left(x^{l} ; 1\right),
$$

and

$$
Q_{2 k}^{S i m}\left(x^{2 l+1} ; 1\right)=0 .
$$




\section{Simpson's Rules for $Q_{0}(f ; h)$}

\subsection{Basic Rule}

We use the Simpson's rule

$$
Q_{0}^{S i m}(f ; h):=\frac{1}{3}[f(-h)+4 f(0)+f(h)]
$$

to approximate $Q_{0}(f ; h)$. The truncation error associated to this process is

$$
\begin{aligned}
R_{0}^{S i m}(f ; h) & :=Q_{0}(f ; h)-Q_{0}^{S i m}(f ; h) \\
& =\frac{1}{h} \int_{-h}^{h} f(x) d x-\frac{1}{3}[f(-h)+4 f(0)+f(h)] .
\end{aligned}
$$

Fromm the preceding properties, $R_{0}^{S i m}\left(x^{l} ; h\right)=h^{l} R_{0}^{S i m}\left(x^{l} ; 1\right)$. By linearity with respect to $f(x)$, the rule is exact for polynomials of degree $\leq 3$ since $R_{0}^{S i m}\left(x^{l} ; 1\right)=0$ for $l=0,1,2,3$. For $l \geq 4$, we also have

$$
\begin{aligned}
R_{0}^{S i m}\left(x^{l} ; 1\right) & =\left(1+(-1)^{l}\right)\left[\frac{1}{l+1}-\frac{1}{3}\right] \\
& =\left\{\begin{array}{cc}
0 & \text { for odd } l \\
2\left[\frac{1}{l+1}-\frac{1}{3}\right] & \text { for even } l
\end{array}\right.
\end{aligned}
$$

\subsection{Corrected Rule}

Let us define the corrected Simpson's rule as follows. For $K=0$ let $Q_{0,0}^{\operatorname{Sim}}(f ; h):=$ $Q_{0}^{\operatorname{Sim}}(f ; h)$, and for $K=1,2, \ldots$, let

$$
\begin{aligned}
Q_{0,2 K}^{\operatorname{Sim}}(f ; h) & :=Q_{0}^{S i m}(f ; h)+\sum_{k=1}^{K} \gamma_{0,2 k} Q_{2 k}(f ; h) \\
& =Q_{0,2(K-1)}^{\operatorname{Sim}}(f ; h)+\gamma_{0,2 K} Q_{2 K}(f ; h)
\end{aligned}
$$

Its truncation error $R_{0,2 K}^{S i m}(f ; h)$ is then defined by $R_{0,0}^{S i m}(f ; h):=R_{0}^{S i m}(f ; h)$ for $K=0$, and by

$$
R_{0,2 K}^{S i m}(f ; h):=R_{0}^{S i m}(f ; h)-\sum_{k=1}^{K} \gamma_{0,2 k} Q_{2 k}(f ; h)
$$




$$
=R_{0,2(K-1)}^{S i m}(f ; h)-\gamma_{0,2 K} Q_{2 K}(f ; h)
$$

for $K=1,2,3, \ldots$ The coefficients $\gamma_{0,2 K}$ are recursively determined by

$$
\gamma_{0,2 K}=\frac{R_{0,2(K-1)}^{S i m}\left(x^{2 K} ; h\right)}{Q_{2 K}\left(x^{2 K} ; h\right)}=\frac{R_{0,2(K-1)}^{S i m}\left(x^{2 K} ; 1\right)}{2(2 K) !},
$$

in such a way that the corrected method is exact for polynomials of degree $2 K$. Moreover, since we have $R_{0,2 K}^{\operatorname{Sim}}\left(x^{2 l+1} ; h\right)=0$ for $l \geq 0$, the method is also exact for polynomials of degree $\leq 2 K+1$.

We observe that $\gamma_{0,2}=0$ because $R_{0,0}^{\operatorname{Sim}}\left(x^{2} ; 1\right)=0$. In fact, from (8) and (9), the $\gamma_{0,2 K}$ 's are recursively defined by the relation

$$
\frac{2}{3}(K-1)=\sum_{k=1}^{K}(2 k) !\left(\begin{array}{c}
2 K+1 \\
2 k
\end{array}\right) \gamma_{0,2 k}
$$

and are related to Bernoulli numbers as follows

$$
\gamma_{0,2 k}=-\frac{4}{3} \frac{\left(4^{k-1}-1\right)}{(2 k) !} B_{2 k}
$$

for $k=1,2, \ldots$, where $B_{2 k}$ is the $2 k$-th Bernoulli numbers [9]. Table 1 contains examples of values of $\gamma_{0,2 k}$.

So we get the following Euler-Maclaurin like formula for the Simpson's rule

$$
\frac{1}{h} \int_{-h}^{h} f(x) d x=Q_{0,2 K}^{S i m}(f ; h)+o\left(h^{2 K+1}\right),
$$

where

$$
\begin{aligned}
& Q_{0,2 K}^{\operatorname{Sim}}(f ; h)=\frac{1}{3}[f(-h)+4 f(0)+f(h)] \\
& \quad+\sum_{k=2}^{K} h^{2 k-1} \gamma_{0,2 k}\left[f^{(2 k-1)}(h)-f^{(2 k-1)}(-h)\right]
\end{aligned}
$$

Let us remark that this formula does not contains the endpoint values of the first derivatives, $f^{(1)}(h)$ and $f^{(1)}(-h)$, of $f(x)$. 


\section{Simpson's Like Rules for $Q_{2}(f ; h)$}

\subsection{Basic Rule}

In an attempt to include values of the first derivatives of $f(x)$ at the endpoints in the corrected formula, we consider the expression

$$
Q_{2}(f ; h):=h\left[f^{(1)}(h)-f^{(1)}(-h)\right]=h \int_{-h}^{h} f^{(2)}(x) d x,
$$

which we would like to approximate as we did for $Q_{0}(f ; h)$ using the nodes of the Simpson's rule. In fact we consider the rule

$$
Q_{2}^{S i m}(f ; h):=2 f(-h)-4 f(0)+2 f(h),
$$

with its truncation error given by

$$
\begin{aligned}
& R_{2}^{S i m}(f ; h):=Q_{2}(f ; h)-Q_{2}^{\operatorname{Sim}}(f ; h) \\
& \quad=h\left[f^{(1)}(h)-f^{(1)}(-h)\right]-[2 f(-h)-4 f(0)+2 f(h)] .
\end{aligned}
$$

We have $R_{2}^{\text {Sim }}\left(x^{l} ; h\right)=h^{l} R_{2}^{\text {Sim }}\left(x^{l} ; 1\right)$. By linearity with respect to $f(x)$, the rule is exact for polynomials of degree $\leq 3$ since $R_{2}^{\text {Sim }}\left(x^{l} ; 1\right)=0$ for $l=0,1,2,3$. For $l \geq 4$, we also have

$$
\begin{aligned}
R_{2}^{\text {Sim }}\left(x^{l} ; 1\right) & =\left(1+(-1)^{l}\right)[l-2] \\
& = \begin{cases}0 & \text { for odd } l, \\
2[l-2] & \text { for even } l .\end{cases}
\end{aligned}
$$

\subsection{Corrected Rule}

Let $Q_{2,0}^{\operatorname{Sim}}(f ; h):=Q_{2}^{\operatorname{Sim}}(f ; h)$, and for $K=1,2, \ldots$, let

$$
\begin{aligned}
Q_{2,2 K}^{\text {Sim }}(f ; h) & \left.=Q_{2}^{\text {Sim }}(f ; h)+\sum_{k=1}^{K} \gamma_{2,2 k} Q_{2 k} f ; h\right) \\
& =Q_{2,2(K-1)}^{\text {Sim }}(f ; h)+\gamma_{2,2 K} Q_{2 K}(f ; h)
\end{aligned}
$$

be the corrected rule. 
The truncation error $R_{2,2 K}^{\operatorname{Sim}}(f ; h)$ is then defined by $R_{2,0}^{\operatorname{Sim}}(f ; h):=R_{2}^{\operatorname{Sim}}(f ; h)$ for $K=0$, and by

$$
\begin{aligned}
R_{2,2 K}^{S i m}(f ; h) & :=R_{2}^{S i m}(f ; h)-\sum_{k=1}^{K} \gamma_{2,2 k} Q_{2 k}(f ; h) \\
& =R_{2,2(K-1)}^{S i m}(f ; h)-\gamma_{2,2 K} Q_{2 K}(f ; h)
\end{aligned}
$$

for $K=1,2,3, \ldots$ The coefficients $\gamma_{2,2 K}^{\operatorname{Sim}}$ are recursively defined by

$$
\gamma_{2,2 K}^{S i m}=\frac{R_{2,2(K-1)}^{S i m}\left(x^{2 K} ; h\right)}{Q_{2 K}\left(x^{2 K} ; h\right)}=\frac{R_{2,2(K-1)}^{S i m}\left(x^{2 K} ; 1\right)}{2(2 K) !}
$$

and the corrected method is exact for polynomials of degree $\leq 2 K$. We also have $R_{2,2 K}^{S i m}\left(x^{2 l+1} ; h\right)=0$ for $l \geq 0$, and form (13) and (14), the method is exact for polynomial of degree $\leq 2 K+1$.

We observe that $\gamma_{2,2}=0$ because $R_{2,0}^{\operatorname{Sim}}\left(x^{2} ; 1\right)=0$, and the $\gamma_{2,2 K}$ 's are recursively defined by the relation

$$
K-1=\frac{1}{2} \sum_{k=1}^{K} \frac{(2 k) !}{2 K+1}\left(\begin{array}{c}
2 K+1 \\
2 k
\end{array}\right) \gamma_{2,2 k} .
$$

Table 1 contains examples of values of $\gamma_{2,2 k}$.

Hence we obtain an Euler-Maclaurin like formula for $Q_{2}(f ; h)$ and $Q_{2}^{S i m}(f ; h)$

$$
h\left[f^{(1)}(h)-f^{(1)}(-h)\right]=Q_{2,2 K}^{\operatorname{Sim}}(f ; h)+o\left(h^{2 K+1}\right),
$$

where

$$
\begin{aligned}
& Q_{2,2 K}^{\operatorname{Sim}}(f ; h)=2 f(-h)-4 f(0)+2 f(h) \\
& \quad+\sum_{k=2}^{K} h^{2 k-1} \gamma_{2,2 k}\left[f^{(2 k-1)}(h)-f^{(2 k-1)}(-h)\right] .
\end{aligned}
$$

\section{A Combination}

\subsection{A Family of Parameter-Dependent Formulae}

We get a family of parameter-dependent formulae by taking a combination of (8) and (13) as follows

$$
\widehat{R}_{2 K, \lambda}^{S i m}(f ; h):=R_{0,2 K}^{S i m}(f ; h)-\lambda R_{2,2 K}^{S i m}(f ; h),
$$




\begin{tabular}{|c||c|c|c|c|}
\hline $2 K$ & 2 & 4 & 6 & 8 \\
\hline$\gamma_{0,2 K}$ & 0 & $-\frac{1}{180}$ & $\frac{1}{1512}$ & $-\frac{1}{14400}$ \\
$\gamma_{2,2 K}$ & 0 & $\frac{1}{12}$ & $-\frac{1}{120}$ & $\frac{17}{20160}$ \\
\hline
\end{tabular}

Table 1: Examples of $\gamma_{0,2 K}$ and $\gamma_{2,2 K}$.

which is exact for polynomials of degree up to $2 K+1$ for any $\lambda$. We get the parameter-dependent Euler-Maclaurin like formula

$$
\frac{1}{h} \int_{-h}^{h} f(x) d x=\widehat{Q}_{2 K, \lambda}^{S i m}(f, h)+o\left(h^{2 K+1}\right),
$$

where

$$
\begin{aligned}
\widehat{Q}_{2 K, \lambda}^{\operatorname{Sim}}(f, h)=Q_{0,2 K}^{\operatorname{Sim}}(f, h)+\lambda R_{2,2 K}^{\operatorname{Sim}}(f ; h) & \\
=\quad & {\left[\left(\frac{1}{3}-2 \lambda\right) f(-h)+\left(\frac{4}{3}+4 \lambda\right) f(0)+\left(\frac{1}{3}-2 \lambda\right) f(h)\right] } \\
& +\sum_{k=1}^{K} h^{2 k-1} \widehat{\gamma}_{2 k}(\lambda)\left[f^{(2 k-1)}(h)-f^{(2 k-1)}(-h)\right],
\end{aligned}
$$

and

$$
\widehat{\gamma}_{2 k}(\lambda)= \begin{cases}\lambda & \text { for } \quad k=1, \\ \gamma_{0,2 k}-\lambda \gamma_{2,2 k} & \text { for } \quad k=2, \ldots, K\end{cases}
$$

\subsection{Increasing Error Order}

To increase the error order we can select $\lambda$ in such a way that (17) be exact also for polynomials of degree up to $2 K+3$. The new expression will contain the endpoint values of the first derivatives $f^{(1)}(x)$ of $f(x), f^{(1)}(-h)$ and $f^{(1)}(h)$, without adding new endpoint values of higher order derivatives of $f(x)$.

Indeed let us consider $\lambda=\lambda_{2 K}$ given by

$$
\lambda_{2 K}=\frac{R_{0,2 K}^{S i m}\left(x^{2(K+1)} ; h\right)}{R_{2,2 K}^{S i m}\left(x^{2(K+1)} ; h\right)}=\frac{R_{0,2 K}^{S i m}\left(x^{2(K+1)} ; 1\right)}{R_{2,2 K}^{S i m}\left(x^{2(K+1)} ; 1\right)} .
$$


This value is well defined if

$$
R_{2,2 K}^{S i m}\left(x^{2(K+1)} ; h\right)=h^{2(K+1)} R_{2,2 K}^{S i m}\left(x^{2(K+1)} ; 1\right) \neq 0 .
$$

But it is easy to show that there exists a polynomial $p_{2 K+2}(x)$ of degree $2 K+2$ such that

$$
\left\{\begin{array}{cl}
p_{2 K+2}(-1)=p_{2 K+2}(0)=p_{2 K+2}(1) & =0 \\
p_{2 K+2}^{(1)}(-1)=-p_{2 K+2}^{(1)}(1) & =1, \\
p_{2 K+2}^{(2 k-1)}(-1)=-p_{2 K+2}^{(2 k-1)}(1) & =0 \text { for } k=2,3,4, \ldots, K,
\end{array}\right.
$$

from which we get $R_{2,2 K}^{S i m}\left(p_{2 K+2}(x) ; 1\right)=2 \neq 0$. This fact implies that

$$
R_{2,2 K}^{S i m}\left(x^{2(K+1)} ; 1\right) \neq 0 .
$$

Finally we obtain the Euler-Maclaurin like formula

$$
\frac{1}{h} \int_{-h}^{h} f(x) d x=\widehat{Q}_{2 K, \lambda_{2 K}}^{\operatorname{Sim}}(f, h)+o\left(h^{2 K+3}\right) .
$$

Examples of values of $\lambda_{2 K}$ are given in Table 2. The corresponding coefficients of the 3-points quadrature rule are given in Table 3 , and the corresponding $\widehat{\gamma}_{2 k}(\lambda)$ are given in Table 4.

\begin{tabular}{|c||c|c|c|c|}
\hline $2 K$ & 2 & 4 & 6 & 8 \\
\hline$R_{0,2 K}^{S i m}\left(x^{2(K+1)} ; 1\right)$ & $-\frac{4}{15}$ & $\frac{20}{21}$ & $-\frac{28}{5}$ & $\frac{1700}{33}$ \\
$R_{2,2 K}^{\operatorname{Sim}}\left(x^{2(K+1)} ; 1\right)$ & 4 & -12 & 68 & -620 \\
$\lambda_{2 K}$ & $-\frac{1}{15}$ & $-\frac{5}{63}$ & $-\frac{7}{85}$ & $-\frac{85}{1023}$ \\
\hline
\end{tabular}

Table 2: Examples of values to compute $\lambda_{2 K}$. 


\begin{tabular}{|c||c||c|c|c|}
\hline $2 K$ & $\lambda_{2 K}$ & $\frac{1}{3}-2 \lambda_{2 K}$ & $\frac{4}{3}+4 \lambda_{2 K}$ & $\frac{1}{3}-2 \lambda_{2 K}$ \\
\hline 2 & $-\frac{1}{15}$ & $\frac{7}{15}$ & $\frac{16}{15}$ & $\frac{7}{15}$ \\
4 & $-\frac{5}{63}$ & $\frac{31}{63}$ & $\frac{64}{63}$ & $\frac{31}{63}$ \\
6 & $-\frac{7}{85}$ & $\frac{127}{255}$ & $\frac{256}{255}$ & $\frac{127}{255}$ \\
8 & $-\frac{85}{1023}$ & $\frac{1533}{3069}$ & $\frac{3072}{3069}$ & $\frac{1533}{3069}$ \\
\hline
\end{tabular}

Table 3: Values of $\lambda_{2 K}$ and the corresponding coefficients of the 3points quadrature rule.

\begin{tabular}{|c|c|c|c|c|}
\hline & \multicolumn{4}{|c|}{$\gamma_{2 k}\left(\lambda_{2 K}\right)$ for $k=1, \ldots, K$} \\
\hline & \multicolumn{4}{|c|}{$2 k$} \\
\hline $2 K$ & 2 & 4 & 6 & 8 \\
\hline 2 & $-\frac{1}{15}$ & & & \\
\hline 4 & $-\frac{5}{63}$ & $\frac{1}{945}$ & & \\
\hline 6 & $-\frac{7}{85}$ & $\frac{1}{765}$ & $-\frac{2}{80325}$ & \\
\hline 8 & $-\frac{85}{1023}$ & $\frac{7}{5115}$ & $-\frac{2}{64449}$ & $\frac{1}{1611225}$ \\
\hline
\end{tabular}

Table 4: Values of $\widehat{\gamma}_{2 k}\left(\lambda_{2 K}\right)$. 


\section{Truncation Error and Taylor's Expansion}

In this section we establish optimal error bounds for appropriate function spaces which allow Taylor's expansions.

\subsection{Function Spaces}

Let $p$ and $q$ be two real numbers such that $1 \leq p, q \leq \infty$ and $\frac{1}{p}+\frac{1}{q}=1$. For an interval $E \subseteq \mathbb{R}$, let $C^{l}(E)$ be the set of continuously differentiable functions up to order $l$ on $E$, and $L^{p}(E)$ be the set of $p$-integrable functions on $E$. Let $A C^{l+1, p}(E)$ be the set of absolutely continuous functions on $E$ defined by : $f \in A C^{l+1, p}(E)$ if and only if

$$
f \in C^{l}(E)
$$

and

$$
\left\{\begin{array}{l}
(a) f^{(l+1)} \in L^{p}(E), \text { and } \\
(b) f^{(l)}(s)=f^{(l)}(r)+\int_{r}^{s} f^{(l+1)}(\xi) d \xi, \forall r, s \in E
\end{array}\right.
$$

\subsection{Taylor's Expansion}

Let $I_{h}=[-h, h]$, for $h=1$ we will simply use $I=[-1,1]$. Taylor's expansion of $f(x) \in A C^{l+1, p}\left(I_{h}\right)$ around $x=0$ of order $l+1$ is

$$
f(x)=\sum_{j=0}^{l} \frac{f^{(j)}(0)}{j !} x^{j}+\int_{-h}^{h} f^{(l+1)}(y) K_{T, l}(x, y ; h) d y
$$

where $K_{T, l}(x, y ; h)$ is the kernel

$$
K_{T, l}(x, y ; h)=\frac{1}{l !}\left[(x-y)_{+}^{l} \mathbf{1}_{[0, h]}(y)+(-1)^{l+1}(y-x)_{+}^{l} \mathbf{1}_{[-h, 0]}(y)\right]
$$

for any $x, y$ in $I_{h}$ (see $\left.[3,10]\right)$. This kernel is a piecewise polynomial function of degree $l$. If we set $x=h \xi$, and $y=h \eta$, then the kernel becomes

$$
K_{T, l}(x, y ; h)=K_{T, l}(h \xi, h \eta ; h)=h^{l} K_{T, l}(\xi, \eta ; 1),
$$

for any $\xi, \eta$ in $I$.

\subsection{Best Error Bounds}

We can now obtain best bounds for these processes. 
Theorem 1. Let us assume that $f(x) \in A C^{n(2 K, \lambda)+1, p}\left(I_{h}\right)$, where $n(2 K, \lambda) \geq$ $2 K$ is the degree of accuracy of $\widehat{R}_{2 K, \lambda}^{\text {Sim }}(f ; h)$. Then there exists a constant $C_{2 K}^{\operatorname{Sim}}(\lambda)$ such that

$$
\left|\widehat{R}_{2 K, \lambda}^{S i m}(f ; h)\right| \leq h^{n(2 K, \lambda)+1-\frac{1}{p}} C_{2 K}^{\operatorname{Sim}}(\lambda)\left\|f^{(n(2 K, \lambda)+1)}\right\|_{p, I_{h}} .
$$

Proof. Since the process is exact for polynomials of degree $\leq n(2 K, \lambda)$, using a Taylor's expansion of order $n(2 K, \lambda)+1$, we obtain

$$
\widehat{R}_{2 K, \lambda}^{S i m}(f ; h)=\int_{-h}^{h} f^{(n(2 K, \lambda)+1)}(y) \widehat{K}_{2 K, \lambda}^{S i m}(y ; h) d y,
$$

where the Peano's kernel $\widehat{K}_{2 K, \lambda}^{S i m}(y ; h)=\widehat{R}_{2 K, \lambda}^{S i m}\left(K_{T, n(2 K, \lambda)}(\cdot, y ; h) ; h\right)$. We observe that

$$
\widehat{K}_{2 K, \lambda}^{S i m}(y ; h)=\widehat{K}_{2 K, \lambda}^{S i m}(h \eta ; h)=h^{n(2 K, \lambda)} \widehat{K}_{2 K, \lambda}^{S i m}(\eta ; 1) .
$$

So

$$
\left\|\widehat{K}_{2 K, \lambda}^{\operatorname{Sim}}(y ; h)\right\|_{q, I_{h}}=h^{n(2 K, \lambda)+1-\frac{1}{p}}\left\|\widehat{K}_{2 K, \lambda}^{\operatorname{Sim}}(\eta ; 1)\right\|_{q, I},
$$

and we get (19) where

$$
C_{2 K}^{\operatorname{Sim}}(\lambda)=\left\|K_{2 K, \lambda}^{\operatorname{Sim}}(\eta ; 1)\right\|_{q, I}
$$

does not depend on $h$.

Since we have

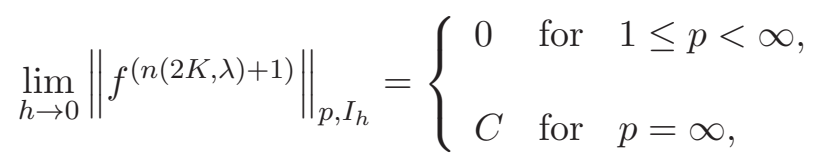

this result says that

$$
\widehat{R}_{2 K, \lambda}^{S i m}(f ; h)= \begin{cases}o\left(h^{n(2 K, \lambda)+1-\frac{1}{p}}\right) & \text { for } 1 \leq p<\infty, \\ O\left(h^{n(2 K, \lambda)+1}\right) & \text { for } p=\infty\end{cases}
$$

Since an $O\left(h^{n(2 K, \lambda)+1-\frac{1}{p}}\right)$ or an $O\left(h^{n(2 K, \lambda)+1}\right)$ is an $o\left(h^{n(2 K, \lambda)}\right)$, means that $\widehat{R}_{2 K, \lambda}^{S i m}(f ; h)=o\left(h^{n(2 K, \lambda)}\right)$.

Remark 2. Using a standard construction $[1,5]$, it can be shown that the bounds given by (19) and (20) are the best bounds. 


\section{Application to other Quadrature Rules}

\subsection{Simpson's $3 / 8$ Rule}

As an example of application to other methods, we can consider the Simpson's $3 / 8$ rule given by

$$
Q_{0}^{S i m}(f ; h):=\frac{1}{4}[f(-h)+3 f(-h / 3)+3 f(h / 3)+f(h)],
$$

and its corresponding expression for the correcting term $Q_{2}(f ; h)$

$$
Q_{2}^{S i m}(f ; h):=\frac{9}{4}[f(-h)-f(-h / 3)+f(h / 3)+f(h)] .
$$

Both expressions are exact for polynomials of degree $\leq 3$, and the approach of this paper can be applied directly (see also [2]).

\subsection{Interpolatory Quadrature Rules}

Simpson's rules are examples of Newton-Cotes formula which are themself examples of interpolatory symmetric quadrature rules. It is clear that the preceding approach can be applied to any other quadrature formula of this type, and the correction might use not only one parameter and $Q_{2}(f ; h)$ but several parameters and expressions $Q_{2 k}(f ; h)$ depending on the degree of accuracy of the basic formula used.

\section{References}

[1] R.A. Adams, Sobolev Spaces, Academic Press, USA (1975).

[2] M.A. Al-Alaoui, A class of numerical integration rules with first order derivatives, $A C M$ SIGNUM Newsletter, 31, No. 2 (1996), 25-44, doi: 10.1145/230922.230930.

[3] E. Asplund and L. Bungart, A first course in integration, Holt, Rinehart and Winston, USA (1966).

[4] P. J. Davis and P. Rabinowitz, Methods of Numerical Integration, 2nd edition, Academic Press, USA (1984), doi: 10.1137/1028042.

[5] F. Dubeau, The method of undetermined coefficients: general approach and optimal error bounds, Journal of Mathematical Analysis, 5, No. 4 (2014), 1-11.

[6] F. Dubeau, On corrected quadrature rules and optimal error bounds, Abstract and Applied Analysis, 2015 (2015), Article ID 461918, 9 pages, doi: 10.1155/2015/461918.

[7] F. Dubeau, On the Euler-Maclaurin formula, Journal of Computational and Applied Mathematics, 296 (2016), 649-660, doi: 10.1016/j.cam.2015.10.023. 
[8] Z. Liu, Error estimate for some composite corrected quadrature rules, Applied Mathematics Letters, 22, No. 5 (2009), 771-775, doi: 10.1016/j.aml.2008.08.016.

[9] D. Sarafyan, L. Derr, and C. Outlaw, Generalizations of the Euler-Maclaurin formula, Journal of Mathematical Analysis and Applications, 67, No. 2 (1979), 542-548, doi: 10.1016/0022-247X(79)90042-8.

[10] L.L. Schumaker, Spline Functions : Basic Theory, Wiley, USA (1981), doi: 10.1137/1.9781611973907. 